\title{
Comunicación y emociones como categorías sociológicas
}

\author{
Communication and Emotions as Sociological Categories
}

Raúl Zamorano

Centro de Estudios Teóricos y Multidisciplinarios en Ciencias Sociales (CETMECS), Facultad de Ciencias

Políticas y Sociales, Universidad Nacional Autónoma de México, México

Karla Hernández

Investigadora independiente, México

RESUMEN

A partir del reconocimiento del carácter autopoiéticamente clausurado del sistema social y de los sistemas psíquicos, analizamos la plausibilidad de ponderar a las emociones en tanto elemento explicativo del orden social y en tanto 'nueva' fuente de conocimiento sobre la sociedad. Con este objetivo, se problematiza el programa de investigación sobre las emociones concebidas como una autodescripción que se ampara en supuestos trascendentales -ontología, retorno al sujeto, intersubjetividady que instala a las emociones tanto en el ámbito de encuentro entre el sujeto y la sociedad cuanto en una dimensión constitutiva de lo social. Si bien las emociones constituyen un presupuesto susceptible de ser tematizado en la comunicación, no pueden fungir como fundamento de la sociedad ni como base de una teoría del conocimiento, aun cuando puedan servir para posicionarse del lado la emoción, con el peligro de seguir moralizando la reflexión.

PAlabRas Clave: Emociones; Comunicación; Sistema psíquico; Sistema social; Conocimiento

\section{ABSTRACT}

Recognizing the autopoietic closure of social and psychic systems, we analyze the plausibility of consider emotions as explanatory basis of social order and as 'new' source of knowledge of society. With this objective, we problematize the research program on emotions conceptualized as a self-description which rest on transcendental postulations - ontology, return to the subject, intersubjectivity- placing the emotions in a field of encounter between subject and society, and as a constitutive dimension of society. Although it is true that emotions are thematizable presuppositions in the communication, these cannot be the basis of society or a knowledge theory, even if they serve to take a stance on the side of emotion, with the risk of turning the reflection into a moral prescription.

KEYWORDS: Emotions; Communication; Psychic system; Social system; Knowledge

\section{INTRODUCCIÓN}

Tal y como nos permite afirmar una larga tradición sociológica, es dable señalar que lo social se explica por lo social y nunca por motivos individuales o por la constitución biológica del cuerpo humano. Sin embargo, en nuestros días, y con el retorno de la sociología de las prácticas, prevalece la idea de que las emociones significan esencialmente importar la explicación científica desde la conceptualización logocéntrica del ser humano, hacia una comprensión universal y verdadera del sujeto. Es como si al mecanismo de las emociones se le asignara un estatuto, un rol ontológico y óntico que debe orientar los procesos no solo de subjetivación sino

\footnotetext{
REVISTA MAD - REVISTA DEL MAGÍSTER EN ANÁLISIS SISTÉMICO APLICADO A LA SOCIEDAD ISSN 0718-0527

Facultad de Ciencias Sociales, Departamento de Antropología.

Universidad de Chile

www.revistamad.uchile.cl
}

DOI: 10.5354/0718-0527.2017.46141 
de conocimiento y coordinación social. Una especie de "dispositivo" (Foucault 1984) objetivado de la subjetividad emotiva, capaz de trasformar la percepción y voluntad particular en una ratio epistémica y así certificar un interés particular a través de un esquema de comportamiento afectivo generalizado. ${ }^{1}$

Es decir, una especie de intersubjetividad del entendimiento/consenso entre actores generada a través de expresiones de significado idéntico y de presentaciones de validez, sobre la base de estructuras lingüísticas transubjetivas que entrelazarían estrechamente entre sí al individuo y la sociedad (Habermas 1989: 448), toda vez que la inclusión en la toma racional de decisiones de la dimensión afectiva viene a confrontar el modelo racional vacío de consideraciones afectivas que promueve el logofalocentrismo capitalista.

En esta lógica, frente a la razón, las emociones son propuestas entonces como una nueva dimensión de lo social que promete explicar las descripciones y la constitución de lo social. Solo que, como el mismo Humberto Maturana reconoce, biológicamente, las emociones son disposiciones corporales que pueden determinar o especificar dominios de acciones; dinamicas corporales que se viven y pueden ser percibidas, pero que en sí mismas no son comunicables, porque incluso la experiencia solo es comunicable en el lenguaje. (Maturana 1994, 2001). ${ }^{2}$

Podemos luego imaginar qué ocurriría si un día la percepción (¿cuál y de quién?) de estas disposiciones corporales, de los comportamientos afectivos (pensemos en el amor), pudiese ser regulada y simbolizada por pretensiones sociales del buen o mal vivir de las emociones.

Por nuestra parte, si bien reconocemos las dificultades que comporta una reflexión sociológica únicamente en el aspecto de la razón, consideramos que, más allá de apelar al lado emoción, una observación sociológica del problema debería preguntarse por la pertinencia de la distinción razón/emoción.

Precisamente con ese objetivo y mediante la utilización de los recursos conceptuales de la Teoría General de los Sistemas Sociales, reflexionaremos sobre las distinciones que orientan a los estudios sobre las emociones $\mathrm{y}$, por lo tanto, evaluaremos las posibilidades reflexivas que abren y las dificultades retóricas que conllevan.

\section{SEMÁNTICA DE LA SUBJETIVIDAD Y COMUNICACIÓN}

Una larga tradición epistémico-metodológica dentro de la ciencia se sustenta en una teoría del conocimiento que está caracterizada por dos su-

\footnotetext{
${ }^{1}$ Una otra ontología de la razón emocional, objetivada paradójicamente contra la razón, a través de una brutal simplificación de la reflexión (eso que Habermas [1990] denomina metafísica)

2 Solo se conocen las emociones cuando se está en el lenguaje, cuando nos movemos en las coordinaciones de coordinaciones de acciones en cualquier dominio que sea; en ese 'lenguajear' que, de hecho, ocurre en la vida cotidiana entrelazado con el emocionar, y donde el lenguaje es el fundamento de todo. No existe ningún elemento fuera del lenguaje, señala Maturana; "lo humano surge, en la historia evolutiva del linaje homínido al que pertenecemos, al surgir el lenguaje" (Maturana 2009: 26).
} 
puestos fundamentales. De un lado, la distinción sujeto/objeto, en donde el conocimiento es entendido como la empresa intencional de un sujeto por aprehender las características verdaderas del objeto externo que observa. De otro, aquella exigencia que intuitivamente parece necesaria, y hasta correcta: el conocimiento solo puede ser conocimiento si se corresponde o refleja el orden de las cosas reales, de lo contrario hablamos de algo diferente.

En esta dirección, y avalada en la autoridad de las teorías de la subjetividad, el pensamiento trascendental sostiene compulsivamente la autoconciencia como fuente espontánea de conocimiento (Spinoza, Husserl). Una posición esencial elevada -casi hegelianamente- a espíritu mismo del conocimiento absoluto y primario del mundo. Al parecer el retorno de esta vieja alianza entre metafísica y oscurantismo, en busca del uno y del todo -Einen und Ganzen (Habermas 1990), del sujeto o del cuerpo perdido, deviene cada vez más preeminente, no obstante, aun cuando opaca y científicamente improductiva.

Si bien es dable suponer, reflexivamente, que los principios de autoconciencia con los cuales los sujetos ordenan un mundo continuo y uniforme no deben de ser tan diferentes unos de otros y que aquello que captamos con nuestros sentidos debe representarse más o menos similarmente en cada individuo, y es plausible también suponer la orientación experiencial de un sujeto observado en estos principios -una episteme epocal (Foucault 1995), ¿podemos hablar de la misma relación de conocimiento sujeto/objeto cuando asumimos la existencia de diferentes sujetos (autoconciencias) cognoscentes?

Es claro que, sobre la base de esta idealización presupuesta, se puede seguir argumentando ('creer') que todos los participantes de una interacción comunicativa perciben y utilizan con idéntico significado las mismas expresiones lingüisticas, sin embargo, si el sujeto ha de retornar a su subjetividad formadora de mundo como horizonte determinante de su auto-interpretación, ¿cómo puede ser constituida una perspectiva de mundo intersubjetivo, donde la intersubjetividad (léase percepciones, emociones) pueda encontrarse con otra intersubjetividad (percepciones, emociones) ${ }^{3}$ no solamente como subjetividad objetivada, para proyectar el mundo? (Habermas 1990, Husserl 1985).

En tal lógica, resulta obvio que el tema de la intersubjetividad, de la búsqueda de refugio en lo totalmente "otro" (Habermas 1990), deviene en un problema precisamente porque no logra problematizar y explicar desde la intersubjetividad situaciones relevantes de la coordinación y orden social (a no ser por la dominación, la cultura en el sentido antropológico, o la supremacía de la razón neoliberal en relación con el cuerpo y las emociones). Es decir, explicar la différence entre construcción de la realidad en

\footnotetext{
${ }^{3}$ Percepción: 1. f. Acción y efecto de percibir. 2. f. Sensación interior que resulta de una impresión material hecha en nuestros sentidos. 3. f. Conocimiento, idea. Emoción: 1. f. Alteración del ánimo intensa y pasajera, agradable o penosa, que va acompañada de cierta conmoción somática. 2. f. Interés expectante con que se participa en algo que está ocurriendo (RAE).
} 
Comunicación y emociones como categorías sociológicas

la psiquis del individuo y la construcción de la realidad en la comunicación social entre estos. Para decirlo de otra manera: la forma en cómo se construye y estabiliza la diferencia entre expectativa psíquica y expectativa social más allá de la percepción o intuición atribuida. Pues, ¿cuál sería la subjetividad del sujeto dentro de la intersubjetividad?, o ¿cuál sería la objetividad del objeto sin una relación de conocimiento?, o ¿basta entonces solo con suponer la realidad empírica de la "intersubjetividad"?

Tal que, y más allá de la lexicografía, ¿que serían socialmente las emociones?

De este modo, se vuelve esencial la necesidad de observar -en la modernidad de la sociedad moderna-lo que significa la posibilidad de la comunicación y de la estabilización de estructuras sociales. Es decir, considerar los criterios y las reglas de conexión que existen en la comunicación para verificar cómo y en qué planos, en un orden policontextual y heterogéneo -característico de la sociedad moderna (Teubner 2005)- estos elementos temporales son plausibles de incorporarse en las comunicaciones de la realidad construida, más allá de las intuiciones, de estados en el mundo (Dasein), de los sujetos (cuerpo) o de los objetos.

Sin embargo, los estudios sobre las emociones son postulados por las ciencias sociales como un aspecto fundamental constitutivo de lo social que pese a su importancia no ha sido debidamente analizado. Lo anterior -se argumenta- debido a que los programas de investigación se han fundamentado en un paradigma tradicional que orienta sus observaciones únicamente al aspecto racional de la sociedad, dejando sin observar lo emocional. Frente al imperio de la palabra, se propone estudiar lo no dicho, aquello que ha sido 'invisibilizado', que está detrás, aquello diverso que se resiste a los ordenamientos unívocos de la razón. ${ }^{4}$

De este modo, nos encontramos frente a un debate que se basa en la oposición razón/emoción, en donde los estudios sobre las emociones vendrían a enfatizan las dificultades que representa un análisis únicamente enfocado a la razón y se pugna por dirigir el análisis hacia el lado de la emoción, como una forma de enfrentar los problemas asociados al estudio exclusivo de la razón. Si bien es claro que tal distinción ha motivado la elaboración de numerosas aportaciones -ya sea inclinándose hacia un lado o al otro-, consideramos pertinente cuestionarnos, ¿cuáles son los rendimientos de la distinción razón/emoción como eje de reflexión en el contexto de las investigaciones sobre la sociedad?

La distinción razón/emoción representa un doble problema si se la quiere proponer como la base de una 'nueva' descripción de la sociedad. Si la emoción se postula como un elemento causal de lo social -aún sin definir claramente qué se entenderá por los términos emoción y sociedad (Turner 2009: 341)- se cae en el problema, ya señalado, de que difícil-

\footnotetext{
${ }^{4}$ El renovado interés en el concepto se constata en un sinnúmero de artículos temáticos: Democracia de las emociones, Emociones de la democracia, Emociones y administración política, Políticas sociales y emociones, Emoción y trabajo, amén de un vasto y largo etcétera.
} 
mente se puede explicar la estabilización de orientaciones sociales (es decir, orden social), debido al carácter altamente contingente y personal de estas. Por otro lado, si se considera a la sociedad como una condición determinante de las emociones individuales (de modo que todo individuo adopte idénticos esquemas emocionales dictados por la sociedad), no podemos explicar la diversidad de formas en las que los individuos pueden experimentar una misma emoción (Le Breton 2013: 71). Por su parte, muchas de las investigaciones en torno al tema de las emociones tratan de resolver ambos riesgos apelando a la existencia de una relación de recíproca influencia entre las emociones y el orden social; sin embargo, hablar de reciprocidad entre la sociedad y la emoción individual oculta el problema de no definir claramente cuál es la relación entre las emociones y la sociedad.

La Teoría General de los Sistemas Sociales (TGSS) nos permite ubicar ambos conceptos en el marco de una teoría de la sociedad. Sin embargo, tendremos que renunciar a comprender a las emociones como parte integrante de lo social y, más bien, reconocer que son el presupuesto de éste. Desde dicha perspectiva, las emociones se inscriben en el ámbito de las operaciones de los sistemas psíquicos, mientras que el orden social sería un atributo del sistema de las comunicaciones.

Esto presupone que tanto los sistemas psíquicos como el sistema social constituyen sistemas autopoiéticamente clausurados, lo cual significa que cada uno de estos opera únicamente mediante sus propias operaciones (Luhmann 2007: 44-45). Los pensamientos constituyen la operación que permite la reproducción de los sistemas psíquicos, mientras que la comunicación es la operación única y necesaria de aquello que llamamos sociedad. En este sentido, el límite operativo entre los sistemas psíquicos y el sistema social (percepción y comunicación) es insuperable, y precisamente en respuesta a esto se construyen formas comunicativas que filtran, vale decir, seleccionan la información y lo que continuará la comunicación, con el objetivo de encontrar cierta seguridad que no se ubica ni en lo emocional ni en lo que se percibe a simple vista (Luhmann 2007).

Es claro que la conciencia percibe y establece diferencias a partir de sí, piensa con sus propios pensamientos, pero no puede dirigir una relación operativa entre conciencia y conciencia, así como la comunicación tampoco es un proceso a partir del cual una conciencia transmite significado, intenciones, emociones o valores a otra conciencia. Por su parte, la comunicación es entendida como la doble distinción desplegada en el tiempo de información/darla-a-conocer/entenderla, y en donde ninguno de estos elementos puede considerarse como principio ontológico frente a los demás, ni siquiera la información precede a la comunicación, ya que incluso la información es una selección que supone comunicación. Asimismo, en este contexto entender no significa interpretar correctamente el sentido de una emisión, sino que simplemente significa observar la distinción información/darla a conocer (Luhmann 2007: 49).

La clausura autopoiética del sistema de comunicaciones y de los 
sistemas de pensamientos implica entonces que ambos sistemas mantienen entre sí una relación del tipo que sostienen un sistema y su entorno. Cuando una distinción es trazada (como en el caso de la distinción sistema/entorno) se generan simultáneamente dos lados, de modo que ni el lado del sistema causa el lado del entorno ni viceversa, sino que ambos son parte de la distinción que les da origen (Luhmann 2007: 41). Ambos lados de la forma se presuponen mutuamente, pues sin sistema no habría entorno y sin entorno no podríamos hablar de sistema.

De este modo, para poder existir, los sistemas psíquicos implican la existencia del sistema social, el cual no determina qué pensamientos deben generarse en la psique. Asimismo, la sociedad como sistema autopoiéticamente clausurado, cuya operación es la comunicación, presupone la existencia de entornos psíquicos para poder operar, sin embargo, los sistemas psíquicos no trasladan sus operaciones (sus pensamientos, ni 'emociones') a las comunicaciones, pues ello significaría romper con la clausura autopoiética que es condición de posibilidad de la sociedad. La comunicación no puede entonces sostenerse, como tradicionalmente se pensaba, en la metafísica de la transmisión de información o en presencia del significado, porque ella escapa del control de la compresión posible de la conciencia del otro (la cual operativamente le es inaccesible). Si, a través de los procesos cognitivos, la conciencia puede procesar como experiencia significativa todo aquello que neurofisiológicamente puede ser captado por las capacidades perceptivas, esto no significa que la experiencia humana pueda tener acceso a la percepción directa, tal cual se le presenta al organismo.

Las conciencias nunca cooperan absolutamente en la comunicación, ya que procesan mucha más información de la que incluso pueden comunicar. En consecuencia, las estructuras de los sistemas sociales tienen autonomía operativa. En última instancia, buscan ante todo aumentar la capacidad de enlace de sus comunicaciones (aun y si es a través de comunicaciones negativas), y no la cooperación o consenso total de las conciencias, muchos menos la integración.

Por ello, en lugar de hablar de integración, optamos por el concepto de acoplamiento estructural. Los acoplamientos estructurales resultan de irritaciones entre los sistemas (sistema psíquico - sistema social) y emanan de las condiciones estructurales de expectativa de éstos, y pueden ser marcadas entonces como una distinción más desde la cual cada sistema puede procesar su información, pero no de las condiciones causales del entorno. Para que la irritación se presente como irritación en el sistema, éste último debe de motivarse a sí mismo a la irritación, independientemente de cómo sea la naturaleza de esa irritación. Deviene entonces importante tener clara la distinción operativa entre la comunicación (sistemas sociales) y los medios de la percepción (sistema psíquico), toda vez que el conocimiento no puede ser reducido a un atributo antropológico del ser humano (percepción, intención, emoción), pues, en primer lugar, este atributo es el sustrato presupuesto de la plausibilidad comunicativa $y$, 
en segundo, precisamente por la condición de simultaneidad del acoplamiento estructural que obliga permanentemente a considerar la distinción operativa conciencia/comunicación, operación/observación (Luhmann 1996: 46s).

Dicho de otra manera, el vínculo que se establece entre el sistema y el entorno ocurre en forma de irritaciones (Luhmann 1996: 34): el sistema en cuestión (ya sea social o psíquico) puede experimentar estímulos provenientes del entorno. Sin embargo, ello no se contrapone con la clausura autopoiética, sino que, por el contrario, la presupone, puesto que toda irritación del entorno es seleccionada siempre desde el sistema. De hecho, usualmente el sistema se permite ignorar la mayoría de las irritaciones que ofrece el entorno (Luhmann 2007: 87).

Como hemos indicado, el sistema mantiene una relación de simultaneidad con el entorno, por lo tanto, el sistema -en tanto existe- se encuentra ya adaptado a este (Luhmann 1996: 27). En el momento en el que un sistema dejara de estar adaptado con respecto a su entorno, simplemente deja de existir como sistema (y, por lo tanto, también se desvanece la distinción sistema/entorno). En consecuencia, resulta incorrecto pensar que las comunicaciones y los pensamientos se orienten hacia estados de mayor (o menor) adaptación/integración entre sí.

Una consecuencia de vital importancia de la distinción sistema/entorno aplicada al caso del sistema social y los sistemas psíquicos es que la clausura autopoiética implica que cada sistema en cuestion, en tanto clausurado, puede generar complejidad interna. Esto debido a que, en el momento en el que se estabiliza un sistema como aupoiéticamente clausurado, este no se ve en la necesidad de corresponder punto por punto con su entorno, sino que todas las relaciones al interior del sistema se generan como operaciones internas sin equivalencia con el exterior. La complejidad de las emociones y de las estructuras sociales es posible precisamente porque no hay coordinación entre ambas.

La clausura autopoiética permite entonces concebir simultáneamente a la sociedad y a los sistemas psíquicos como unidades sin tener por ello que renunciar a la diversidad de formas posibles que implica cada una. De este modo, la sociedad y los sistemas psíquicos coexisten como entidades descargadas de las determinaciones de sus respectivos entornos: las emociones y los pensamientos individuales pueden ser diversos y completamente "desadaptados" de aquello dictado por la sociedad a la vez que la sociedad puede generar estructuras propias sin la necesidad de una correspondencia con todo aquello que ocurre al interior de los sistemas psíquicos.

En términos operativos, la manera en que cada uno de nosotros constituye un mundo de la experiencia siempre genera diferencias en la observación perceptual o, por al menos, deviene imposible saber si es verdad que todos, apelando a la pura neurofisiología, percibimos uno y el mismo mundo, ya que la única manera de acceder a lo percibido por el organismo es a través del despliegue de operaciones cognitivas de la con- 
Comunicación y emociones como categorías sociológicas

ciencia que empero permanecen desconocidas si no se comunican. Entonces, ¿cómo podemos deducir que las capacidades perceptivas de otra conciencia perceptiva son similares a las mías, si no podemos penetrar la conciencia de los otros?

Es con el uso del lenguaje que se hace patente la intención comunicativa, evitando la comprensión o atribución a la acción del sujeto, pues queda clara la distinción entre el acto de comunicar y la información (a diferencia de los comportamientos o conductas perceptibles: emociones). Tal que, para la observación desde la teoría de sistemas, es determinante una diferencia y no un objeto unitario. El medio coordina esa diferencia y la forma lo concretiza. Determinadas formas pueden generalizarse y servir, más tarde, como sustratos mediales, las cuales, en el decurso evolutivo, logran imponerse a los medios, pero siempre como reutilización constante de las formas como medios. Esto implica operativamente que las emociones como un sustrato medial no comunican algo determinado como tal, pues no se trata de una relación de coincidencia entre el pensamiento y el ser o el sistema psíquico y el entorno, sino de una maleabilidad de sí mismo, una autoestructuración del sistema (Luhmann 1996: 139) que -como señalamos- solo puede ser conocida si se comunica. $Y$ aquí ya no importa la totalidad de las emociones reconocidas como correlato de la percepción, sino la manera en que se procesan comunicativamente las situaciones cognitivas como una diferencia.

Toda operación de percepción, observación y comunicación está asimismo necesariamente temporalizada, ${ }^{5}$ toda vez que siempre se puede preguntar quién actualiza y bajo qué condiciones. Es decir, está delimitada por los marcos semánticos resultantes de la articulación del espacio de experiencia y por el horizonte de expectativas disputados en el acontecimiento comunicativo (Koselleck 1993: 205-357). Con esto además, como señala Maturana (2001), la biología de la percepción también se ve modificada con la distinción que se pone a disposición para la observación (la distinción sujeto/objeto), tal que el sistema de referencia humano/cuerpo/masa biológica (sujeto) resulta entonces una designación demasiado amplia y demasiado estrecha de la observación y del proceso de producción del conocimiento. Estrecha porque el ser humano hace más que solo observar y amplia porque no hay manera de que el proceso de reproducción de percepciones y conocimiento sea procesado por una conciencia individual (lo cual está igualmente más allá de nuestras capacidades cognitivas).

Teniendo esto en cuenta, es posible observar que las emociones no pueden conformar el eje de reflexion sobre el cual se sustenten investigaciones sobre la sociedad. Por supuesto, las emociones pueden ser tema de

\footnotetext{
${ }^{5}$ Luhmann (1996: 79) señala que toda permanencia de objetos tiene que ser construida con base en diferencias temporales, y eso solo puede ocurrir operativamente en observaciones actuales en cada caso, que, en el momento de surgir, ya están desapareciendo. Incluso el hecho de que eso sea así, de que cada acontecimiento por lo tanto esté insertado en un antes inactual y un después inactual y que solo es actualizado como de paso, incluso esto puede ser observado únicamente de esta manera.
} 
la comunicación, pero operativamente siguen formando parte del entorno de la sociedad. Y, como hemos señalado, tal conceptualización sistémica que a primera vista podría considerarse "reduccionista", posibilita observar tanto a la sociedad como a la individualidad psíquica, cada una en toda su diversidad y complejidad. Mientras que, por otro lado, postular a las emociones como una dimensión constitutiva de lo social lleva al investigador a posicionarse de un lado u otro de la distinción razón/emoción y a construir argumentos que apelen a la determinación de la razón por la emoción o de la emoción por la razón (con las consecuencias que ya hemos mencionado) o a esbozar de manera poco clara la existencia de formas de reciprocidad entre ambos lados.

Concebir, además, a las emociones como origen y frontera del acto de conocer, ahonda el abismo en el subjetivismo voluntarista sobre bases axiológicas y potencia el planteamiento de que la evolución de la sociedad se puede predecir mediante idénticas emociones o ritos que gobiernan y definen el curso de ésta.

Es por esto que las emociones difícilmente podrían fungir como un concepto explicativo rector para describir fenómenos sociales; sin embargo, un programa de investigación que reflexione sobre las emociones en tanto comunicaciones sería compatible con reflexiones que conceptualicen a la sociedad como un sistema autopoiéticamente clausurado. ${ }^{6}$ Una vía para ello sería considerar las emociones, no como la operación constitutiva de lo social sino como parte de las observaciones que la sociedad ha generado sobre sí misma, es decir, como una semántica ${ }^{7}$ que resulta cada vez menos plausible en la sociedad funcionalmente diferenciada.

\section{ORDEN SOCIAL Y EMOCIONES}

En este punto, se puede señalar que la forma de operar de la sociedad moderna pone de relieve con particular claridad las dificultades que el concepto de emoción enfrenta para erigirse como una categoría explicativa en la construcción del orden social, pues la despersonalización y desacoplamiento espacio-temporal típicos de la sociedad funcionalmente diferenciada otorgan oportunidades para la tematización de las emociones mucho más restringidas de lo que presumen los estudios que reivindican el papel 'psicosocial' de las emociones. Ejemplificaremos esta posición mediante el caso de los Medios de Comunicación Simbólicamente Generalizados (en adelante $\mathrm{MCSG}$ ).

Tal y como ya se ha mencionado, toda comunicación opera de modo ortogonal con respecto a la operatividad de los sistemas psíquicos,

\footnotetext{
${ }^{6} \mathrm{Al}$ menos algunos autores, que trabajan el tema, reconocen a las emociones como fenómenos que necesariamente han de ser comunicados para poder ser estudiados. Al respecto consultar la Revista Latinoamericana de estudios sobre Cuerpos, Emociones y Sociedad 2010, núm. 4.

7 Las semánticas son estructuras que orientan observaciones posteriores y recuerdan aquellas distinciones que considera dignas de ser conservadas, al mismo tiempo que deja caer en el olvido otras distinciones (Luhmann 2007: 425).
} 
sin embargo, la operatividad clausurada que distingue entre comunicaciones y emociones se observa claramente en la forma de operación de los MCSG. En el proceso evolutivo desde formas de diferenciación premodernas a la forma de diferenciación funcional, la sociedad ha perdido la posibilidad de regirse a sí misma desde un centro específico, tal como otrora fue la religión y la moral (Luhmann 2007: 178). En lugar de tener un ámbito de coordinación central, la sociedad moderna ha desarrollado MCSG para enfrentar y desarrollar problemas específicos de modo particular.

Los MCSG evolucionaron como medios orientados cada uno por un código unitario que excluye terceros valores (Luhmann 2007: 280s), por ejemplo, el código legal/no-legal, sano/enfermo y verdad/no-verdad, entre otros. Mediante la utilización del código, cada MCSG se encuentra claramente diferenciado de la operación de los demás medios, sin embargo, todos comparten la característica de enfrentar la improbabilidad de la comunicación motivando a la aceptación (o rechazo) de determinadas comunicaciones (Luhmann 2007: 156). La estabilización de un código implica que todas las comunicaciones generadas como parte del medio en cuestión se orientarán únicamente hacia un lado o hacia el otro de la forma. En el caso del medio de la verdad (que posibilita la reproducción del sistema de la ciencia), por ejemplo, toda comunicación que forme parte de dicho medio debe orientarse por el código verdad/no verdad. De este modo, queda claro que la selectividad de la comunicación es tan alta que resulta poco plausible hablar de la influencia de las emociones en las comunicaciones de dichos sistemas expertos. Y aún en el caso de que las emociones fueran tematizadas en la comunicación, éstas dejarían de ser emociones en el mismo momento en el que fueran comunicadas.

Más aún, el surgimiento de los MCSG, cuyo funcionamiento como tales caracteriza a la sociedad moderna, supuso el desarrollo de la escritura y, por lo tanto, la superación de la co-presencia, de la comunicación cara a cara como modalidad única y preeminente en la sociedad. De este modo, los MCGS se han desacoplado del tiempo y del espacio (Luhmann 2007: 198). Se han desanclado (Giddens 2004: 31) y operan más allá del carácter contextual y co-presencial que presupone e implica la emoción.

Evidentemente, todo MCSG presupone la operatividad de sistemas de conciencia (Luhmann 2007: 295), sin embargo, la forma de dos lados del código motiva únicamente a la oscilación entre ambos lados de la forma, de modo que -por ejemplo- en el medio de la verdad solo es posible aceptar o rechazar comunicaciones orientadas por el código verdad/no verdad. A partir de que se han estabilizado estos códigos de preferencia, habrá que elegir entre un lado y el otro, generando una alta selectividad con respecto a otras comunicaciones y, por supuesto, con respecto a todas las operaciones que tengan lugar dentro de la psique: las emociones resultan entonces irrelevantes al momento de oscilar en el medio de la verdad, si bien pueden ser tematizadas como un problema para el sistema de la ciencia, tal y como se hace en la presente comunicación científica. 
Los conceptos y categorías que se usan en el sistema de la ciencia, como todo sistema experto, son observables a pesar de que las situaciones varíen, siempre y cuando respondan a los principios de la construcción programática y sus definiciones sean claras (selección conceptualizada) al interior del mismo sistema científico. Ello porque toda constitución de un problema de investigación es, principalmente, una reducción de complejidad interna de posibilidades desde donde se puede abordar dicho objeto (una construcción reflexiva que tiene claridad comparativa).

Nuevamente, la conciencia no puede ser el sustento operativo del conocimiento social, porque eso nos llevaría a pensar que la conciencia es el sustento de la comunicación; de la misma manera que el sistema nervioso central no puede ser sustento operativo de la conciencia: el sistema nervioso solo está capacitado para discriminar los estados orgánicos propios del organismo (internos, sin referencia al entorno). Esto significa que la conciencia está capacitada para distinguir entre lo interno del cuerpo de lo externo, a pesar de que éste último se le presente como externo, pues aparece como objeto de la conciencia, posibilitando construir un mundo a partir del cual puede hacer estas distinciones (cuerpo/conciencia y mundo interno/mundo externo) e inclusive observarse a sí misma. Sin embargo, como hemos señalado, la conciencia es inasible para la comunicación porque la percepción es incomunicable (solo la comunicación es comunicable).

Ciertamente, el mundo construido por la conciencia es imaginación intuitiva nacida de la percepción orgánica (imaginario radical), pero esto por sí mismo no guarda un sentido que pueda ser comunicado. Solo una red de comunicación que construye el sentido posible de la comunicación de la percepción hace posible la comunicación de la percepción (de emociones). Además, es claro que en la percepción aprehendemos lo diverso, pero también de manera diversa, como unidad. Es decir, lo distintivo desaparece en la esencia misma de la cosa. En cambio, en la comunicación se procesa la distinción como distinción. Especialmente como una distinción entre información y dar-a-conocer, solo que no a través de otras percepciones sino y solo a través de otras comunicaciones.

No es la percepción la que genera estas posibilidades y estructuras, es con la comunicación que se construyen maneras a partir de las cuales se pueden coordinar los sistemas psíquicos, aun cuando esto no asegure una cooperación absoluta de los sistemas entre sí o la coordinación absoluta entre sistemas y entorno, más bien hace plausible solo la conexión con otras comunicaciones. Así, en la estructuración del sistema social (comunicación), la conciencia -entorno- le sirve como filtro y como vínculo con los entornos físicos, químicos y biológicos, los cuales no perturban ni irritan a la primera. Solo se hace plausible que el sistema psíquico, por medio del acoplamiento con el lenguaje, logre que las percepciones, intereses o emociones, puedan encontrar un canal dentro de la comunicación (Luhmann 1996: 38). 
En esta perspectiva, la actividad cognoscitiva necesaria para la experiencia no intuye, percibe o manipula las "cosas en sí" para luego obtener propiedades que luego se ven reflejadas en la conciencia del agente, sino que organiza internamente las experiencias a partir de las cuales se puede constituir un mundo accesible y en el que su complejidad se ve reducida (operaciones que posibilitan operaciones). Y si bien este proceso se realiza en un nivel pre-lingüístico -pues las operaciones cognitivas de la conciencia humana, e incluso de su neurofisiología, no están supeditadas al lenguaje- hay una diferencia abismal entre el percibir y el comunicar una percepción (emoción). En primer lugar, porque la restricción del tiempo para realizar una comunicación (y más sobre la percepción) es un imperativo del proceso comunicativo. Es decir, no hay manera de comunicar lo que se percibe al momento que se percibe, porque entre la percepción como un momento del organismo y la comunicación de la percepción como un momento social, hay un proceso de filtrado infranqueable. ${ }^{8}$ En segundo lugar, esta diferencia radica en que la información comunicada pueda ser entendida por aquel al que se le comunica. Esto es, independientemente del estado real de la conciencia perceptiva al momento de percibir o al momento de configurar lingüísticamente la percepción, aun cuando exista la posibilidad de que el otro participante de la comunicación decodifique y comprenda la información compartida en un sentido muy específico, a saber, que el primero comunica sobre su experiencia del mundo $\mathrm{y}$, por lo tanto, que tiene capacidades perceptivas comparativamente similares (porque el otro tambien experimenta al mundo).

Sin embargo, el nivel de la percepción experiencial se vuelve a complejizar cuando no solo se busca comunicarla, sino además cuando se constituye como criterio de validez para aceptar el conocimiento. Esto significa que la referencia a las capacidades sensoriales fisiológicas puede ajustar la comunicación sobre aquello que presupone como entorno, ${ }^{9}$ pero en caso alguno es el determinante para asignar los valores de verdad del conocimiento. Porque específicamente, qué sería entonces una emoción, ¿solo aquella reconocida como experiencia práctica relevante o como absoluto de quien la percibe?

Reconociendo que la percepción es una manera más de obtener información, también es relevante mostrarse de acuerdo en que la constitu-

\footnotetext{
${ }^{8}$ Del organismo a la conciencia y de la conciencia a la comunicación se requieren varios procesos de acoplamiento estructural: en este sentido elemental, cada sistema opera simultáneamente con su entorno y por lo tanto tiene que presuponer que en el entorno sucede algo a lo cual solo se podrá reaccionar en el siguiente paso, y que solo se puede influenciar en el futuro. El tiempo del acoplamiento estructural por lo tanto está ordenado analógicamente, mientras que todo conocimiento se presenta digitalmente, siendo que, en consecuencia, toda seriación causal -relativa al entorno o también al sistema mismo- requiere una transformación de analogicidad en digitalidad. Por eso no sabemos qué es el tiempo en sí, sino que solo podemos utilizar diferenciaciones relativas al tiempo (por ejemplo, antes/después), cuando tiene sentido presentar el mundo como ordenado en el sentido temporal (Luhmann 1996: 122).

${ }^{9}$ En tanto ruido, el sistema puede o no ignorar las percepciones del entorno, pero es el propio sistema el que define cuáles percepciones son relevantes y para qué situaciones. Esto lo lleva a precisamente a reaccionar ante la comunicación de percepciones, siempre y cuando sean relevantes para orientar un análisis reflexivo sobre la percepción (Luhmann 1996: 167).
} 
ción de conocimiento en la ciencia responde a un tipo especial de espacio de experiencia y de horizonte de expectativas, lo cual significa que están explícitamente constituidos a través de conceptos y no de una correspondencia con una realidad allá afuera (trascendente), independiente de nosotros. Si científicamente observamos conceptos, entendidos como expectativas comunicativas condensadas, entonces su precisión teórica es de vital importancia para asegurar precisamente la cientificidad de sus observaciones.

\section{EPÍTOME}

Resulta vacuo tratar de sustituir la razón con la emoción y, a partir de la percepción, intentar inducir -sea desde el margen de racionalidad que sea- la generalización de las emociones. Como semántica es posible observar que el programa de investigación sobre las emociones acierta en señalar que la sociedad no puede ya explicarse en términos de 'racionalidad'. Sin embargo, el límite de esta observación se encuentra en que no permite superar o replantearse la pertinencia de la distinción razón/emoción. Además, si en el devenir civilizatorio se generan socialmente semánticas y estructuras comunicativas como una guía que hace plausible confiar en la inconsolable realidad del vivir juntos (orden social), lo anterior no implica que exista correspondencia alguna con todo aquello que ocurre al interior de cada sistema psíquico. De igual forma, implica reconocer que emociones y pensamientos individuales son también completamente diversos de aquello que comunicativamente se estructura como pensamiento y emoción.

Sabido es que evolutivamente toda sociedad está soportada por su propia memoria autoproducida - la cual interviene en todas las operaciones mediante comunicaciones. Comunicaciones que los participantes dan precisamente por conocida, por familiar, tanto en el plano social cuanto en el plano psíquico, lo cual les permite orientarse, pero de manera alguna les determina. En esta perspectiva y parafraseando a Niklas Luhmann, las emociones son precisamente el presupuesto sobre el cual el sistema psíquico percibe, diferencia y construye sentidos que luego, a través del acoplamiento con el lenguaje, comunica.

$\mathrm{Al}$ respecto, una fascinante reflexión en la forma como sociedad y sistemas psíquicos coexisten en tanto entidades descargadas de las determinaciones de sus entornos, nos la ofrece Gunther Teubner (2005) en su análisis del clásico de García Márquez, Crónica de una muerte anunciada.

De acuerdo con el autor, lo fascinante es precisamente que, y sobre el presupuesto de la emotividad de todos los involucrados, el homicidio igual se lleva a cabo, porque y más allá de las emociones, la comunicación sobre un compromiso social no oficial orienta -por sobre la sanción legalla motivación y el sentido -no solo- de los hermanos Vicario. Incluso las emociones (sentidos, motivaciones internas), cuyo basamento está fundado en valores y sentimientos de honra, no logran articularse convin- 
centemente para impedir un crimen que nadie desea. Lo observable es más bien la permanente tensión (auto-irritación) entre el sistema psíquico (conciencia) y el sistema social (comunicación). Lo mismo podemos advertir en la novela Abril quebrado (Kadaré 2001), en donde el código social formal (contrato de sangre) termina por orientar la lógica comunicativa más allá del río heraclitiano de emociones que fluyen en el transcurso de la historia. Interesante que la literatura devenga reflexiva y analíticamente más seductora que la investigación 'psicosocial' sobre las emociones

Resulta evidente que la conciencia, pudiendo irritar a la comunicación, no puede determinarla, así como la comunicación tampoco puede determinar a la conciencia. De allí que no pueda existir algo como intersubjetividad que conecte a las conciencias entre sí, que las entrelace, que las amalgame y que esta quimera pueda conducir a la existencia de significados idénticos (Luhmann 1996: 11-14). Tal que, y aun reconociendo que las emociones no se suprimen en forma alguna, es importante también tener presente que en tanto emociones permanecen en la conciencia de sus creadores y dejan de ser emociones en el momento que se comunican, para luego ser imputables o imputadas precisamente como comunicaciones

Entender los acoplamientos y desacoplamientos que se dan entre sistemas psíquicos y sistemas sociales a la hora de enmarcar las percepciones y emociones obliga entonces a preguntarse al menos qué relación se puede observar entre emoción y comunicación, si estas en sí construyen estructuras sociales, y -reconociendo que el conocimiento no puede ser reducido a un atributo antropológico del ser humano- preguntarse cómo pueden ser estas construidas y por parte de quién (Luhmann 2015: 132), sobre todo cuando las características de la sociedad moderna trascienden la oposición emoción/razón, lo cual reclama precisamente una conceptualización puntual y más amplia que contemple las formas en que la referencia a la experiencia o a la percepción, como criterio de asignación de validez del conocimiento científico, es cognoscitivamente accesible al sistema de la ciencia (como comunicación codificada), mas no como supuesto trascendental o metafísico.

Las ciencias sociales tienen entonces que poner especial cuidado en la argumentación de sus construcciones objetuales, tal que la novedad no sea tan insólita como para que sea incapaz de enlazarse operativa y reflexivamente ${ }^{10}$ ni que la repetición sea el permanente apelo a trozos de teoría que imbrican cuerpo, emociones y sociedad en un eterno retorno a lo absoluto y trivial. RM

\footnotetext{
${ }^{10}$ Un sistema es reflexivo cuando está en condiciones para afirmar su autonomía observando su identidad en la medida en que se convierte en accesible a sí mismo. Es decir, mediante la aplicación de la comunicación a la comunicación, de la reflexión a la reflexión y no a la cosa (Luhmann 1991; Luhmann 2007: 105).
} 


\section{REFERENCIAS}

Foucault, M. (1984). Saber y verdad. Madrid: Ediciones de la Piqueta.

Foucault, M. (1995). Las palabras y las cosas. México DF: Siglo XXI Editores.

Giddens, A. (2004). Consecuencias de la modernidad. Madrid: Alianza editorial.

Habermas, J. (1989). El discurso filosófico de la modernidad. Madrid: Taurus.

Habermas, J. (1990). Pensamiento postmetafísico. México DF: Taurus.

Habermas, J. (2002). Teoría de la acción comunicativa I. Racionalidad de la acción y racionalidad social. México DF: Taurus.

Husserl, E. (1985). Meditaciones cartesianas. México DF: Fondo de Cultura Económica.

Kadaré, I. (2001). Abril quebrado. Madrid: Alianza Editorial.

Koselleck, R. (1993). Futuro pasado. Barcelona: Editorial Paidós.

Le Breton, D. (2013). Por una antropología de las emociones. Revista latinoamericana de estudios sobre cuerpos, emociones y sociedad, 10, 69-79.

Luhmann, N. (1991). Sistemas sociales. Lineamientos para una teoría general. México DF: Alianza Editorial.

Luhmann, N. (1996). La ciencia de la sociedad. México DF: Editorial AnthroposUniversidad Iberoamericana-Iteso.

Luhmann, N. (2007). La sociedad de la sociedad. México DF: Editorial Herder.

Luhmann, N. (2015). Comunicaciones y cuerpo en la teoría de los sistemas sociales. México DF: Casa Editora La Biblioteca - UNAM.

Maturana, H. (1994). El ojo del observador. Barcelona: Gedisa.

Maturana, H. (2001). Emociones y lenguaje en educación y política. Santiago de Chile: Dolmen.

Maturana, H. (2009). Realidad: ¿objetiva o construida? México DF: AnthroposUniversidad Iberoamericana-ITESO.

Teubner, G. (2005). Direito, sistema e policontexturalidade. São Paulo: Editora UNIMEP.

Turner, J. (2009). The Sociology of Emotions: Basic Theoretical Arguments. Emotion Review, 1(4), 340-354.

SOBRE LOS AUTORES

Raúl Zamorano es Profesor-investigador del Centro de Estudios Teóricos y Multidisciplinarios en Ciencias Sociales de la Universidad Nacional Autónoma de México y miembro del Sistema Nacional de Investigadores (CONACYT). Doctor en Filosofía Jurídica por el Centro di Studi Sul Rischio, Università di Lecce, Italia.

Karla Hernández es Licenciada en Sociología por la Universidad Nacional Autónoma de México. Actualmente cursa maestría en la Universidad Autónoma Metropolitana (UAM - Cuajimalpa).

\section{CONTACTO}

Universidad Nacional Autónoma de México (UNAM),

Facultad de Ciencias Políticas y Sociales

Circuito Mario de La Cueva s/n, Ciudad Universitaria, 04510 Coyoacán,

México DF, México

karla199007@hotmail.com

Recibido: diciembre 2016

Aceptado: abril 2017 